PROCEEDINGS OF THE

AMERICAN MATHEMATICAL SOCIETY

Volume 131, Number 12, Pages 3633-3641

S 0002-9939(03)07186-7

Article electronically published on July 9, 2003

\title{
ON ALTERNATING ANALOGUES OF TORNHEIM'S DOUBLE SERIES
}

\author{
HIROFUMI TSUMURA
}

(Communicated by Wen-Ching Winnie Li)

\begin{abstract}
In this paper, we give some evaluation formulas for alternating analogues of Tornheim's double series. These can be regarded as alternating analogues of Mordell's formulas. This gives a partial answer to the problem posed by Subbarao-Sitaramachandrarao.
\end{abstract}

\section{INTRODUCTION}

Tornheim considered the double series $T(p, q, r)$ defined by

$$
T(p, q, r)=\sum_{m, n=1}^{\infty} \frac{1}{m^{p} n^{q}(m+n)^{r}}
$$

where $p, q, r$ are nonnegative integers with $p+r>1, q+r>1$ and $p+q+r>2$ (see [5]). He showed that $T(p, q, N-p-q)$ is a polynomial in $\{\zeta(j) \mid 2 \leq j \leq N\}$ with rational coefficients when $N$ is odd and $N \geq 3$ (see also [2]).

In [3, Mordell gave an evaluation formula for $T(2 k, 2 k, 2 k)$ for a positive integer $k$. Furthermore, in [4], Subbarao and Sitaramachandrarao generalized Mordell's formula, and considered alternating analogues of (1.1) defined by

$$
\begin{aligned}
& R(p, q, r)=\sum_{m, n=1}^{\infty} \frac{(-1)^{n}}{m^{p} n^{q}(m+n)^{r}}, \\
& S(p, q, r)=\sum_{m, n=1}^{\infty} \frac{(-1)^{m+n}}{m^{p} n^{q}(m+n)^{r}} .
\end{aligned}
$$

They posed the problem to evaluate $R(p, p, p)$ and $S(p, p, p)$ for any positive integer $p$. As a partial answer to their problem, we gave an evaluation formula for $S(p, p, p)$ for any positive odd integer $p$ (see [6], Corollary 3).

The purpose of this paper is to give an evaluation formula for $R(p, p, p)$ for any odd positive integer $p$ with $p \geq 3$ (see Theorem 3.6). In order to prove this formula,

Received by the editors March 22, 2002 and, in revised form, May 28, 2002.

2000 Mathematics Subject Classification. Primary 11M06; Secondary 30B99, 33E20, 40A05, 40B05.

Key words and phrases. Tornheim's double series, Euler numbers, Riemann's zeta function, uniformly convergent series.

(C)2003 American Mathematical Society 
we make use of the same method as we introduced in [6]. Indeed, we consider partial Tornheim's double series

$$
\mathfrak{T}_{b_{1}, b_{2}}(p, q, r)=\sum_{m, n=0}^{\infty} \frac{1}{\left(2 m+b_{1}\right)^{p}\left(2 n+b_{2}\right)^{q}\left(2 m+2 n+b_{1}+b_{2}\right)^{r}},
$$

where $b_{1}, b_{2} \in\{1,2\}$. As a result, we can write $\mathfrak{T}_{1,1}(p, p, q)$ as a rational linear combination of products of Riemann's zeta values at positive integers, when $p$ and $q$ are odd positive integers with $q \geq 3$ (see Proposition 3.5).

More general results on partial Tornheim's double series defined by (1.4) will be given in [7].

The author wishes to express his sincere gratitude to the referee who gave him valuable comments and carefully pointed out the errors in this paper.

\section{Preliminaries}

Let $\mathbb{N}$ be the set of natural numbers, $\mathbb{N}_{0}=\mathbb{N} \cup\{0\}, \mathbb{Z}$ the ring of rational integers, and $\mathbb{R}$ the field of real numbers. Let $i=\sqrt{-1}$. Throughout this paper we fix $\delta \in \mathbb{R}$ with $\delta>0$. For $u \in \mathbb{R}$ with $u \in[1,1+\delta]$ and $s \in \mathbb{R}$, we define $\rho(s ; u):=\sum_{m \geq 0}(-u)^{-m} /(2 m+1)^{s}$. If $u>1$, then $\rho(s ; u)$ is convergent for any $s \in \mathbb{Z}$. Let $\rho(s):=\rho(s ; 1)$. We define a set of numbers $\left\{\mathcal{E}_{m}(u)\right\}$ by

$$
F(x ; u)=\frac{2 u e^{x}}{e^{2 x}+u}=\sum_{m=0}^{\infty} \mathcal{E}_{m}(u) \frac{x^{m}}{m !} .
$$

Note that $\left\{\mathcal{E}_{m}(1)\right\}$ are the Euler numbers (see, e.g., [1]). So we have $\mathcal{E}_{2 j+1}(1)=$ $0\left(j \in \mathbb{N}_{0}\right)$. It follows from (2.1) that if $u \in[1,1+\delta]$, then

$$
\liminf _{m \rightarrow \infty}\left(\frac{\left|\mathcal{E}_{m}(u)\right|}{m !}\right)^{-1 / m} \geq \frac{\pi}{2}
$$

From the relation $F(x ; u)=2 \sum_{n \geq 0}(-u)^{-n} e^{(2 n+1) x}$, we obtain the following.

Lemma 2.1. For $k \in \mathbb{N}_{0}$ and $u \in(1,1+\delta]$,

$$
\rho(-k ; u)=\frac{1}{2} \mathcal{E}_{k}(u) .
$$

For $r \in \mathbb{N}, p \in \mathbb{N}_{0}, u \in[1,1+\delta]$ and $\theta \in \mathbb{R}$, we define

$$
\mathcal{X}_{p}(\theta, r ; u):=\sum_{n=0}^{\infty} \frac{(-u)^{-n} \sin ^{(p)}((2 n+1) \theta)}{(2 n+1)^{r}},
$$

where we denote the $l$-th derivative of a function $f(\theta)$ by $f^{(l)}(\theta)$. Using the wellknown relation

$$
\sin ^{(p)}(\theta)=\frac{i^{p-1}}{2}\left(e^{i \theta}+(-1)^{p-1} e^{-i \theta}\right)=i^{p-1} \sum_{n=0}^{\infty} \lambda_{p+1+n} \frac{(i \theta)^{n}}{n !},
$$

where $\lambda_{j}:=\left(1+(-1)^{j}\right) / 2$, we have

$$
\mathcal{X}_{p}(\theta, r ; u)=i^{p-1} \sum_{m=0}^{\infty} \rho(r-m ; u) \lambda_{p+1+m} \frac{(i \theta)^{m}}{m !}
$$


when $u \in(1,1+\delta]$. By (2.2) and (2.3), we see that (2.6) is uniformly convergent with respect to $u \in(1,1+\delta]$ when $\theta \in(-\pi / 2, \pi / 2)$. Furthermore we define

$$
\mathcal{Y}_{p}(\theta, r ; u):=\mathcal{X}_{p}(\theta, r ; u)-i^{p-1} \sum_{j=0}^{r} \rho(r-j ; u) \lambda_{p+1+j} \frac{(i \theta)^{j}}{j !},
$$

for $r \in \mathbb{N}, p \in \mathbb{N}_{0}, u \in[1,1+\delta]$ and $\theta \in \mathbb{R}$. When $u \in(1,1+\delta]$,

$$
\mathcal{Y}_{p}(\theta, r ; u)=i^{p-1} \sum_{n=1}^{\infty} \rho(-n ; u) \lambda_{p+1+n+r} \frac{(i \theta)^{n+r}}{(n+r) !} .
$$

This is also uniformly convergent with respect to $u \in(1,1+\delta]$ when $\theta \in(-\pi / 2, \pi / 2)$. So it follows from Lemma 2.1 and the fact $\mathcal{E}_{2 j+1}(1)=0\left(j \in \mathbb{N}_{0}\right)$ that

$$
\mathcal{Y}_{r}(\theta, r ; u) \rightarrow 0 \quad(u \rightarrow 1 ; r \in \mathbb{N}, \theta \in(-\pi / 2, \pi / 2)) .
$$

Now we define

$$
\begin{aligned}
\mathfrak{S}(k, s ; u) & :=\sum_{m, n=0}^{\infty} \frac{(-u)^{-m-n}}{\{(2 m+1)(2 n+1)\}^{2 k+1}(2 m+2 n+2)^{s}}, \\
\mathfrak{R}(k, s ; u) & :=\sum_{m, n=0}^{\infty} \frac{(-u)^{-2 m-n-1}}{\{(2 m+1)(2 m+2 n+3)\}^{2 k+1}(2 n+2)^{s}},
\end{aligned}
$$

for $k \in \mathbb{N}_{0}, s \in \mathbb{Z}, u \in[1,1+\delta]$. By an elementary calculation just the same as that in Lemma 3 of [6], we obtain the following.

Lemma 2.2. For $k \in \mathbb{N}_{0}, u \in(1,1+\delta]$ and $\theta \in \mathbb{R}$,

$$
\begin{aligned}
& \sum_{m=0}^{\infty} \frac{(-u)^{-m} e^{i(2 m+1) \theta}}{(2 m+1)^{2 k+1}} \cdot \sum_{n=0}^{\infty} \frac{(-u)^{-n} e^{i(2 n+1) \theta}}{(2 n+1)^{2 k+1}}=\sum_{m=0}^{\infty} \mathfrak{S}(k,-m ; u) \frac{(i \theta)^{m}}{m !}, \\
& \sum_{m=0}^{\infty} \frac{(-u)^{-m} e^{-i(2 m+1) \theta}}{(2 m+1)^{2 k+1}} \cdot \sum_{n=0}^{\infty} \frac{(-u)^{-n} e^{i(2 n+1) \theta}}{(2 n+1)^{2 k+1}} \\
& \quad=\sum_{m=0}^{\infty} \mathfrak{R}(k,-m ; u)\left\{1+(-1)^{m}\right\} \frac{(i \theta)^{m}}{m !}+\sum_{m=0}^{\infty} \frac{u^{-2 m}}{(2 m+1)^{4 k+2}} .
\end{aligned}
$$

For $n \in \mathbb{Z}, k \in \mathbb{N}_{0}$ and $u \in(1,1+\delta]$, we define

$$
\begin{aligned}
\beta_{n}(k ; u):= & \frac{1}{2}\left\{\mathfrak{S}(k,-n ; u)+\left(1+(-1)^{n}\right) \mathfrak{R}(k,-n ; u)\right\} \\
& -\sum_{\nu=0}^{k}\left(\begin{array}{c}
n \\
2 \nu
\end{array}\right) \rho(2 k+1-2 \nu ; u) \rho(2 k+1+2 \nu-n ; u) .
\end{aligned}
$$

In particular when $n \leq-1$, we define $\beta_{n}(k ; 1)$ by $(2.13)$ with $u=1$. By combining (2.13) and Lemma 2.2, we obtain the following.

Lemma 2.3. For $k \in \mathbb{N}_{0}, u \in(1,1+\delta]$ and $\theta \in \mathbb{R}$,

$$
\begin{aligned}
& \mathcal{Y}_{2 k+1}(\theta, 2 k+1 ; u) \sum_{n=0}^{\infty} \frac{(-u)^{-n} e^{i(2 n+1) \theta}}{(2 n+1)^{2 k+1}} \\
& \quad=(-1)^{k} \sum_{n=0}^{\infty} \beta_{n}(k ; u) \frac{(i \theta)^{n}}{n !}+\frac{(-1)^{k}}{2} \sum_{m=0}^{\infty} \frac{u^{-2 m}}{(2 m+1)^{4 k+2}} .
\end{aligned}
$$


Proof. By (2.5) and Lemma 2.2, we have

$$
\begin{aligned}
& \mathcal{X}_{2 k+1}(\theta, 2 k+1 ; u) \sum_{n=0}^{\infty} \frac{(-u)^{-n} e^{i(2 n+1) \theta}}{(2 n+1)^{2 k+1}} \\
& =\frac{i^{2 k}}{2} \sum_{n=0}^{\infty}\left\{\mathfrak{S}(k,-n ; u)+\left(1+(-1)^{n}\right) \mathfrak{R}(k,-n ; u)\right\} \frac{(i \theta)^{n}}{n !} \\
& +\frac{(-1)^{k}}{2} \sum_{m=0}^{\infty} \frac{u^{-2 m}}{(2 m+1)^{4 k+2}} .
\end{aligned}
$$

On the other hand, by combining (2.6) and

$$
\sum_{n=0}^{\infty} \frac{(-u)^{-n} e^{i(2 n+1) \theta}}{(2 n+1)^{2 k+1}}=\sum_{n=0}^{\infty} \rho(2 k+1-n ; u) \frac{(i \theta)^{n}}{n !},
$$

we have

$$
\begin{aligned}
& \left\{i^{2 k} \sum_{j=0}^{2 k+1} \rho(2 k+1-j ; u) \lambda_{j} \frac{(i \theta)^{j}}{j !}\right\} \sum_{n=0}^{\infty} \frac{(-u)^{-n} e^{i(2 n+1) \theta}}{(2 n+1)^{2 k+1}} \\
& =(-1)^{k} \sum_{n=0}^{\infty} \sum_{\nu=0}^{k}\left(\begin{array}{c}
n \\
2 \nu
\end{array}\right) \rho(2 k+1-2 \nu ; u) \rho(2 k+1+2 \nu-n ; u) \frac{(i \theta)^{n}}{n !} .
\end{aligned}
$$

By (2.13), we obtain the proof.

Since (2.7) and (2.15) are uniformly convergent with respect to $u \in(1,1+\delta]$ when $\theta \in(-\pi / 2, \pi / 2)$ by $(2.2)$, so is $(2.14)$, and

$$
\liminf _{m \rightarrow \infty}\left(\frac{\left|\beta_{m}(k ; u)\right|}{m !}\right)^{-1 / m} \geq \frac{\pi}{2}
$$

for $k \in \mathbb{N}_{0}$. Furthermore, by (2.8), we have

$$
\begin{aligned}
& \lim _{u \rightarrow 1} \beta_{m}(k ; u)=0 \quad(m \in \mathbb{N}), \\
& \lim _{u \rightarrow 1} \beta_{0}(k ; u)=-\frac{1}{2} \sum_{m=0}^{\infty} \frac{1}{(2 m+1)^{4 k+2}} .
\end{aligned}
$$

\section{Evaluation Formulas}

By (2.13), we have

$$
\begin{aligned}
\beta_{2 j+1}(k ; u)= & \frac{1}{2} \mathfrak{S}(k,-2 j-1 ; u) \\
& -\sum_{\nu=0}^{k}\left(\begin{array}{c}
2 j+1 \\
2 \nu
\end{array}\right) \rho(2 k+1-2 \nu ; u) \rho(2 k+2 \nu-2 j ; u),
\end{aligned}
$$

for $j \in \mathbb{N}_{0}$. 
Lemma 3.1. For $k, l \in \mathbb{N}_{0}, p \in\{0,1\}, u \in(1,1+\delta]$ and $\theta \in \mathbb{R}$,

$$
\begin{aligned}
& \frac{1}{2} \sum_{m, n=0}^{\infty} \frac{(-u)^{-m-n} \sin ^{(p)}((2 m+2 n+2) \theta)}{\{(2 m+1)(2 n+1)\}^{2 k+1}(2 m+2 n+2)^{2 l+p}} \\
& -\sum_{\nu=0}^{k} \rho(2 k+1-2 \nu ; u) \sum_{\tau=0}^{2 \nu}\left(\begin{array}{c}
2 l+p-1+2 \nu-\tau \\
2 l+p-1
\end{array}\right) \frac{(-\theta)^{\tau}}{\tau !} \\
& \cdot \mathcal{X}_{\tau+p}(\theta ; 2 k+2 l+1+p+2 \nu-\tau ; u) \\
& =i^{p-1} \sum_{j=-l-p}^{\infty} \beta_{2 j+1}(k ; u) \frac{(i \theta)^{2 j+2 l+1+p}}{(2 j+2 l+1+p) !} .
\end{aligned}
$$

Proof. By (2.5) and (2.9), we have

$$
\begin{aligned}
\sum_{m, n=0}^{\infty} & \frac{(-u)^{-m-n} \sin ^{(p)}((2 m+2 n+2) \theta)}{\{(2 m+1)(2 n+1)\}^{2 k+1}(2 m+2 n+2)^{2 l+p}} \\
& =i^{p-1} \sum_{m=-2 l-p}^{\infty} \mathfrak{S}(k,-m ; u) \lambda_{m+1} \frac{(i \theta)^{m+2 l+p}}{(m+2 l+p) !}
\end{aligned}
$$

On the other hand, we use (2.5) and consider the function $f(x ; d, \theta)=\sin ^{(p)}(x \theta) x^{-d}$ in the argument of Lemma 6 of [6]. Then we obtain that

$$
\begin{aligned}
& \sum_{\tau=0}^{r}\left(\begin{array}{c}
d-1+r-\tau \\
d-1
\end{array}\right) \frac{(-\theta)^{\tau}}{\tau !} \frac{\sin ^{(\tau+p)}(\theta x)}{x^{d+r+q-\tau}} \\
& =i^{p-1} \sum_{m=-d}^{\infty}(-1)^{r}\left(\begin{array}{c}
m \\
r
\end{array}\right) \frac{(i \theta)^{m+d}}{(m+d) !} \lambda_{p+1+m+d} x^{-q-r+m},
\end{aligned}
$$

by using the well-known relation $\left(\begin{array}{c}-X \\ j\end{array}\right)=(-1)^{j}\left(\begin{array}{c}X+j-1 \\ j\end{array}\right)$. Putting $r=2 \nu, q=2 k+1$ and $d=2 l+p$, we have

$$
\begin{aligned}
& \sum_{\nu=0}^{k} \rho(2 k+1-2 \nu ; u) \sum_{\tau=0}^{2 \nu}\left(\begin{array}{c}
2 l+p-1+2 \nu-\tau \\
2 l+p-1
\end{array}\right) \frac{(-\theta)^{\tau}}{\tau !} \\
& \cdot \mathcal{X}_{\tau+p}(\theta ; 2 k+2 l+1+p+2 \nu-\tau ; u) \\
& =i^{p-1} \sum_{m=-2 l-p}^{\infty} \sum_{\nu=0}^{k}\left(\begin{array}{c}
m \\
2 \nu
\end{array}\right) \rho(2 k+1-2 \nu ; u) \rho(2 k+1+2 \nu-m ; u) \\
& \cdot \lambda_{m+1} \frac{(i \theta)^{m+2 l+p}}{(m+2 l+p) !}
\end{aligned}
$$

Put $m=2 j+1$. Then, by (3.1), we obtain the proof.

By (2.16), we can let $u \rightarrow 1$ in both sides of (3.2) when $l \in \mathbb{N}$ and $\theta \in[-\pi / 2, \pi / 2]$. By (2.17), we obtain the following. 
Proposition 3.2. For $k \in \mathbb{N}_{0}, l \in \mathbb{N}, p \in\{0,1\}$ and $\theta \in[-\pi / 2, \pi / 2]$,

$$
\begin{aligned}
& \frac{1}{2} \sum_{m, n=0}^{\infty} \frac{(-1)^{m+n} \sin ^{(p)}((2 m+2 n+2) \theta)}{\{(2 m+1)(2 n+1)\}^{2 k+1}(2 m+2 n+2)^{2 l+p}} \\
& -\sum_{\nu=0}^{k} \rho(2 k+1-2 \nu) \sum_{\tau=0}^{2 \nu}\left(\begin{array}{c}
2 l+p-1+2 \nu-\tau \\
2 l+p-1
\end{array}\right) \frac{(-\theta)^{\tau}}{\tau !} \\
& \cdot \mathcal{X}_{\tau+p}(\theta ; 2 k+2 l+1+p+2 \nu-\tau ; 1) \\
& =i^{p-1} \sum_{j=-l-p}^{-1} \beta_{2 j+1}(k ; 1) \frac{(i \theta)^{2 j+2 l+1+p}}{(2 j+2 l+1+p) !} .
\end{aligned}
$$

For simplicity, we let $\psi(s):=\sum_{m>0} 1 /(2 m+1)^{s}=\left(1-2^{-s}\right) \zeta(s)$ for $s>1$, where $\zeta(s)$ is the Riemann zeta function. It is well-known that $\sin ^{(2 j)}((2 m+1) \pi / 2)=$ $(-1)^{j+m}$ and $\sin ^{(2 j+1)}((2 m+1) \pi / 2)=0$ for $j, m \in \mathbb{N}_{0}$. Hence $\mathcal{X}_{2 j}(\pi / 2, s ; 1)=$ $(-1)^{j} \psi(s)$ and $\mathcal{X}_{2 j+1}(\pi / 2, s ; 1)=0$. Putting $p=0, \theta=\pi / 2$ and $l=m+1$ for $m \in \mathbb{N}_{0}$ in (3.3), we have

$$
\begin{gathered}
\sum_{r=0}^{m} \beta_{2 r-2 m-1}(k ; 1) \frac{(i \pi / 2)^{2 r+1}}{(2 r+1) !} \\
=-i \sum_{\nu=0}^{k} \rho(2 k+1-2 \nu) \sum_{\eta=0}^{\nu}\left(\begin{array}{c}
2 m+1+2 \nu-2 \eta \\
2 m+1
\end{array}\right) \\
\cdot \frac{(i \pi / 2)^{2 \eta}}{(2 \eta) !} \psi(2 k+2 m+3+2 \nu-2 \eta),
\end{gathered}
$$

for $k \in \mathbb{N}_{0}$. We recall the following lemma which can be obtained by replacing $\pi$ with $\pi / 2$ in Lemma 8 of [6].

Lemma 3.3. Suppose $\left\{P_{m}\right\}$ and $\left\{Q_{m}\right\}$ are sequences which satisfy the relation

$$
\sum_{j=0}^{m} P_{m-j} \frac{(i \pi / 2)^{2 j+1}}{(2 j+1) !}=Q_{m}
$$

for any $m \in \mathbb{N}_{0}$. Then the relation

$$
P_{m}=\frac{2}{i \pi} \sum_{\nu=0}^{m}\left(1-2^{2 \nu+1-2 m}\right) 2^{2 \nu+1-2 m} \zeta(2 m-2 \nu) Q_{\nu}
$$

holds for any $m \in \mathbb{N}_{0}$. Note that $\zeta(0)=-\frac{1}{2}$.

By (3.4), we can apply Lemma 3.3 with $P_{m}=\beta_{-2 m-1}(k ; 1)$ and $Q_{m}=-i \mathcal{Z}_{0}(k, m)$ for $m \in \mathbb{N}_{0}$, where

$$
\begin{aligned}
\mathcal{Z}_{p}(k, m):= & \sum_{\nu=p}^{k} \rho(2 k+1-2 \nu) \sum_{\eta=0}^{\nu-p}\left(\begin{array}{c}
2 m+1-2 p+2 \nu-2 \eta \\
2 m+1-p
\end{array}\right) \\
\cdot \psi(2 k+2 m-2 p+3+2 \nu-2 \eta) & \frac{(-1)^{\eta}(\pi / 2)^{2 \eta+p}}{(2 \eta+p) !}
\end{aligned}
$$

for $p \in\{0,1\}$. Then we have

$$
\beta_{-2 m-1}(k ; 1)=-\frac{2}{\pi} \sum_{\nu=0}^{m}\left(1-2^{2 \nu+1-2 m}\right) 2^{2 \nu+1-2 m} \zeta(2 m-2 \nu) \mathcal{Z}_{0}(k, \nu),
$$


for $m \in \mathbb{N}_{0}$.

Remark 3.4. It follows from (2.2) that both sides of

$$
\sum_{m=0}^{\infty} \frac{(-u)^{-m} \cos ((2 m+1) \pi / 2)}{(2 m+1)^{2 k+1}}=\sum_{n=0}^{\infty} \rho(2 k+1-2 n ; u) \frac{(i \pi / 2)^{2 n}}{(2 n) !}
$$

are uniformly convergent with respect to $u \in(1,1+\delta]$, when $k \in \mathbb{N}$. Letting $u \rightarrow 1$, we have

$$
\sum_{j=0}^{k} \rho(2 k+1-2 j) \frac{(i \pi / 2)^{2 j}}{(2 j) !}=0,
$$

because $\rho(-2 m-1 ; u) \rightarrow 0\left(u \rightarrow 1 ; m \in \mathbb{N}_{0}\right)$. Hence we can see that if $k \in \mathbb{N}$, then

$$
\begin{array}{r}
\mathcal{Z}_{p}(k, m)=\sum_{\nu=1}^{k} \rho(2 k+1-2 \nu) \sum_{\eta=0}^{\nu-1}\left(\begin{array}{c}
2 m+1-2 p+2 \nu-2 \eta \\
2 m+1-p
\end{array}\right) \\
\cdot \psi(2 k+2 m-2 p+3+2 \nu-2 \eta) \frac{(-1)^{\eta}(\pi / 2)^{2 \eta+p}}{(2 \eta+p) !}
\end{array}
$$

for $p \in\{0,1\}$.

Now we can prove the following result on $\mathfrak{T}_{1,1}(2 k+1,2 k+1,2 l+1)$ defined by (1.4).

Proposition 3.5. For $k \in \mathbb{N}_{0}$ and $l \in \mathbb{N}$,

$$
\begin{gathered}
\mathfrak{T}_{1,1}(2 k+1,2 k+1,2 l+1) \\
=-2 \mathcal{Z}_{1}(k, l)+\frac{4}{\pi} \sum_{m=0}^{l} \sum_{\nu=0}^{m}\left(1-2^{2 \nu+1-2 m}\right) 2^{2 \nu+1-2 m} \zeta(2 m-2 \nu) \\
\cdot \mathcal{Z}_{0}(k, \nu) \frac{(i \pi / 2)^{2 l-2 m}}{(2 l-2 m) !} .
\end{gathered}
$$

Proof. We put $p=1$ and $\theta=\pi / 2$ in (3.3). Since $\cos ((2 m+2 n+2) \pi / 2)=$ $(-1)^{m+n+1}$, we have

$$
-\frac{1}{2} \mathfrak{T}_{1,1}(2 k+1,2 k+1,2 l+1)=\mathcal{Z}_{1}(k, l)+\sum_{m=0}^{l} \beta_{-2 m-1}(k ; 1) \frac{(i \pi / 2)^{2 l-2 m}}{(2 l-2 m) !} .
$$

By (3.6), we obtain the proof.

Finally we prove an evaluation formula for $R(2 k+1,2 k+1,2 k+1)$ for any $k \in \mathbb{N}$. By (1.1), (1.2) and (1.4), we can see that

$$
\begin{aligned}
& R(2 k+1,2 k+1,2 k+1) \\
& \quad=2^{-6 k-3} T(2 k+1,2 k+1,2 k+1)-\mathfrak{T}_{1,1}(2 k+1,2 k+1,2 k+1),
\end{aligned}
$$

for $k \in \mathbb{N}_{0}$. It was proved that

$$
T(2 k+1,2 k+1,2 k+1)=-4 \sum_{j=0}^{k}\left(\begin{array}{c}
4 k-2 j+1 \\
2 k
\end{array}\right) \zeta(2 j) \zeta(6 k-2 j+3)
$$

(see [2], Eq. (1.14)). By combining (3.9), (3.10) and Proposition 3.5, we obtain the following. 
Theorem 3.6. For $k \in \mathbb{N}$,

$$
\begin{aligned}
& R(2 k+1,2 k+1,2 k+1) \\
& =-2^{-6 k-1} \sum_{j=0}^{k}\left(\begin{array}{c}
4 k-2 j+1 \\
2 k
\end{array}\right) \zeta(2 j) \zeta(6 k-2 j+3)+2 \mathcal{Z}_{1}(k, k) \\
& -\frac{4}{\pi} \sum_{m=0}^{k} \sum_{\nu=0}^{m}\left(1-2^{2 \nu+1-2 m}\right) 2^{2 \nu+1-2 m} \zeta(2 m-2 \nu) \mathcal{Z}_{0}(k, \nu) \frac{(i \pi / 2)^{2 k-2 m}}{(2 k-2 m) !}
\end{aligned}
$$

where

$$
\begin{array}{r}
\mathcal{Z}_{p}(k, m)=\sum_{\nu=1}^{k} \rho(2 k+1-2 \nu) \sum_{\eta=0}^{\nu-1}\left(\begin{array}{c}
2 m+1-2 p+2 \nu-2 \eta \\
2 m+1-p
\end{array}\right) \\
\cdot \psi(2 k+2 m-2 p+3+2 \nu-2 \eta) \frac{(-1)^{\eta}(\pi / 2)^{2 \eta+p}}{(2 \eta+p) !}
\end{array}
$$

for $p \in\{0,1\}$. Note that $\rho(s)=\sum_{m \geq 0}(-1)^{m} /(2 m+1)^{s}$ and $\psi(s)=\left(1-2^{-s}\right) \zeta(s)$.

Example 3.7. We list several evaluation formulas for $R(2 k+1,2 k+1,2 k+1)$ deduced from Theorem 3.6. Note that we use the relations

$$
\rho(2 j+1)=\frac{(-1)^{j} E_{2 j}}{2(2 j) !}\left(\frac{\pi}{2}\right)^{2 j+1} \quad\left(j \in \mathbb{N}_{0}\right),
$$

where $\left\{E_{n}\right\}$ are the Euler numbers (see, e.g., [1]).

$$
\begin{aligned}
R(3,3,3)= & \frac{253}{256} \pi^{2} \zeta(7)-\frac{2545}{256} \zeta(9) \\
R(5,5,5)= & \frac{2039}{18432} \pi^{4} \zeta(11)+\frac{285565}{24576} \pi^{2} \zeta(13)-\frac{2056257}{16384} \zeta(15) \\
R(7,7,7)= & \frac{32639}{2211840} \pi^{6} \zeta(15)+\frac{913913}{491520} \pi^{4} \zeta(17)+\frac{40212403}{262144} \pi^{2} \zeta(19) \\
& -\frac{896163411}{524288} \zeta(21) \\
R(9,9,9)= & \frac{522239}{275251200} \pi^{8} \zeta(19)+\frac{2978549}{66060288} \pi^{6} \zeta(21)+\frac{1194884977}{41943040} \pi^{4} \zeta(23) \\
& +\frac{71693105055}{33554432} \pi^{2} \zeta(25)-\frac{1625043751045}{67108864} \zeta(27) .
\end{aligned}
$$

Remark 3.8. More general results on partial Tornheim's double series $\mathfrak{T}_{b_{1}, b_{2}}(p, q, r)$ defined by (1.4) will be given in [7. Indeed, we will be able to give more general relation formulas for $\mathfrak{T}_{b_{1}, b_{2}}(p, q, r)$.

\section{REFERENCES}

1. K. Dilcher, Zeros of Bernoulli, generalized Bernoulli and Euler polynomials, Memoirs of Amer. Math. Soc. 73, No. 386 (1988). MR 89h:30005

2. J. G. Huard, K. S. Williams and Nan-Yue Zhang, On Tornheim's double series, Acta Arith. 75-2 (1996), 105-117. MR 97f:11073

3. L. J. Mordell, On the evaluation of some multiple series, J. London Math. Soc. 33 (1958), 368-371. MR 20:6615

4. M. V. Subbarao and R. Sitaramachandra Rao, On some infinite series of L. J. Mordell and their analogues, Pacific J. Math. 119 (1985), 245-255. MR 87c:11091

5. L. Tornheim, Harmonic double series, Amer. J. Math. 72 (1950), 303-314. MR 11:654a 
6. H. Tsumura, On some combinatorial relations for Tornheim's double series, Acta Arith. 105 (2002), 239-252.

7. H. Tsumura, On Tornheim's type of double harmonic series, submitted.

Department of Management Informatics, Tokyo Metropolitan College, Akishima, TOKYO 196-8540 JAPAN

E-mail address: tsumura@tmca.ac.jp 